\title{
Atrial Fibrillation Analysis for Real Time Patient Monitoring
}

\author{
Ragheed Allami ${ }^{1}$, Andrew Stranieri ${ }^{1}$, Faezeh Marzbanrad ${ }^{2}$, Venki Balasubramanian ${ }^{1}$, Herbert F. \\ Jelinek $^{3}$ \\ ${ }^{1}$ Centre for Informatics and Applied Optimisation, Federation University, Victoria, Australia \\ ${ }^{2}$ School of Electrical and Computer Systems Engineering, Monash University, Victoria, Australia \\ ${ }^{3}$ School of Community Health, Charles Sturt University, NSW, Australia
}

\begin{abstract}
Atrial Fibrillation (AF) can lead to life-threatening conditions such as stroke and heart failure. The instant recognition of life-threatening cardiac arrhythmias based on a 3-lead ECG to record a Lead II configuration for a few seconds is a challenging problem of clinical significance. Five consecutive ECG beats that were identified by a cardiologist to characterise an AF episode and five consecutive heartbeat intervals representing an irregular RR intervals episode were analysed. The detection and analysis of $P$ waves as the morphological features of $A F$ was executed based on two template matching methods. An AF detector was developed by combining the correlation coefficients based on the template matching methods and the standard deviation of the RR intervals. The AF detector was then applied to classify 5 consecutive beats as AF or non-AF based on thresholding the calculated irregularity.

The proposed algorithm was tested on the MIT-BIH Atrial Fibrillation and the Challenge 2017 databases. The proposed method resulted in an improved sensitivity, specificity and accuracy of 97.60\%, 98.20\% and 99\% respectively compared to recent published methods. In addition, the proposed method is suitable for real-time patient monitoring as it is computationally simple and requires only a few seconds of ECG recording to detect an AF rhythm.
\end{abstract}

\section{Introduction}

An Electrocardiogram (ECG) records the electrical impulses of the heart and indicates rhythm anomalies for diagnostic purposes [1]. A typical ECG tracing of the cardiac cycle consists of a $\mathrm{P}$ wave, QRS complex, and $\mathrm{T}$ wave [2]. The performance of an ECG analysis system depends upon the accurate and reliable detection of the QRS complex, as well as the $\mathrm{T}$ and $\mathrm{P}$ waves [3].

Atrial Fibrillation (AF) are irregular heartbeats caused by obstruction along electrical impulse pathways of the heart atria hence [4]. AF is linked to fast uncoordinated atrial fluctuations where the contraction rate may exceed 600 beats per minute. Hence, it often leads to a sudden vanishing of $\mathrm{P}$-waves, which is usually linked with synchronous functioning of the atria. $\mathrm{P}$ waves are substituted by an oscillating baseline that is composed of fibrillatory wavelets of low amplitude.

$\mathrm{AF}$ represents one of the most common chronic cardiovascular arrhythmias, appearing in $2 \%$ of the population globally [4]. AF is linked with stroke, heart failure, risk of death and coronary artery disease and found to be connected with significant morbidity and mortality $[4,5]$. Approximately 12 million North Americans and Europeans suffer from $\mathrm{AF}$ and this is estimated to triple in the next 30 to 50 years [6]. The prevalence of AF rises with age. It is affecting $0.5 \%$ of people at the age between 40 and 50 years and $5-15 \%$ at the age of 80 years [7]. The automated detection and classification of AF based on a simple and fast method over a few heartbeats is important for prompt, accurate diagnosis and treatment to reduce morbidity and mortality.

Figure 1 illustrates typical ECG diagnostic features in a segment of normal rhythm including the distance between $R$ waves called the $R R$ interval. $R R$ intervals in normal rhythms are regular as shown in figure 1 whereas in AF rhythms, they are irregular as demonstrated in figure 2 . Therefore, many studies described below have used RR intervals to detect $\mathrm{AF}$.

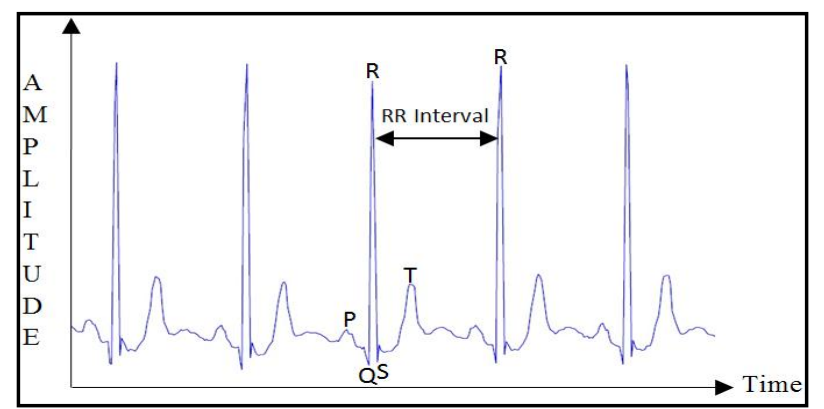

Figure 1. A segment of Normal ECG for Record No. A00823 readings from Challenge 2017 database showing $\mathrm{P}, \mathrm{QRS}$ and T and RR interval. 


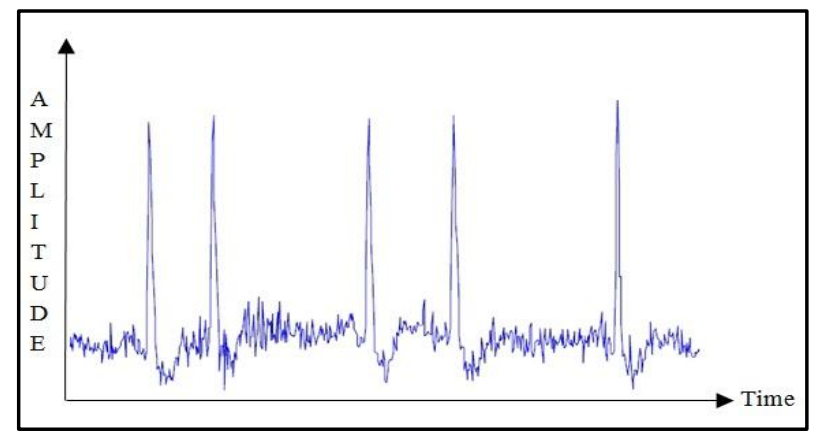

Figure 2. A segment of AF rhythm for Record No. 06995 reading from MIT-BIH Atrial Fibrillation database.

From figure 2, it is apparent that RR intervals are irregular and most $\mathrm{P}$ waves are absent or have low amplitude. Accordingly, numerous investigations harnessed these two key points to classify and detect AF.

$\mathrm{AF}$ detection methods can be grouped under ventricular function analysis-based and atrial function analysis-based approaches. Classification based on ventricular function analysis using RR intervals is the most common method in real-time studies to detect AF [8]. For example, RR data that was generated from a 30 second trace, was employed to classify AF using statistical detrended fluctuation analysis (DFA), the local dynamics score (LDs) and sample entropy (COSEn). The results indicated that the positive predictive values for $\mathrm{AF}$ were $97 \%$ [8]. In addition, A low complexity method introduced by Petrenas et al. [9] based on statistical techniques of RR data from a sliding window of 8 beats was utilized to discern AF from normal rhythms. The results for sensitivity and specificity were $97.1 \%$ and $98.3 \%$ respectively. Moreover, Zhou et al. [10] presented a novel approach using Shannon entropy and symbolic dynamics with sensitivity, specificity and accuracy values were $96.14 \%, 95.73 \%$, 95.97\% respectively.

Combining both ventricular and atrial features could plausibly achieve improved performance by including ventricular and atrial features to detect $\mathrm{AF}$ disease. Babaeizadeh et al. [11] employed a Markov modeling technique to compute irregular RR intervals and two morphology features P and PR intervals were then applied to three statistical classifiers to detect AF rhythms with $92 \%$ sensitivity and $97 \%$ specificity. A four features-based classification included RR intervals, $P$ wave absence, $f$ wave presence and noise level and submitted to a fuzzy logic classifier to detect AF episodes. The fuzzy logic classifier provided sensitivity, specificity and accuracy of $100 \%, 76 \%$ and $88 \%$ respectively. Other techniques have been applied to classify AF rhythm including nonlinear complexity measures, wavelet transform and artificial neural networks such as described in [12-15] However, these techniques may not always be technically feasible for real-time processing of ECG data due to the requirement of high computational resources [16].

Brief periods, usually less than 30 seconds, play an important role in cardiac research to detect AF rhythm [17]. Recent investigations have recommended that the use of short time intervals such as five seconds may be sufficient for the detection of AF due to increased risk of stroke [17-19]. Real time, continuous cardiac monitoring platforms have emerged that utilize wearable ECG sensors and smartphones for biosignal interpretation. However, these devices have limited power, memory and processor capacity and are not suitable for implementation of complex AF classification algorithms as data streaming through these devices consumes considerable power resources. Hence, computationally simple and fast algorithms based on ventricular and atrial features that can accurately detect an AF rhythm from only a few ECG signals is crucial.

The method advanced here applies two templates to identify the presence or absence of $\mathrm{P}$ waves. The standard deviation of normal to normal RR cycles is computed to find the irregular RR data then two simple rules are employed to detect an AF episode from non-AF.

The algorithms are described in the materials section next before experimental results are reported.

\section{Materials}

Trials of the AF detection algorithm advanced here were applied to the MIT-BIH Atrial Fibrillation (MIT-BIH AF) database [20] for testing and the Challenge 2017 database [21] was employed for measuring the generalization capability performance of the algorithm. The MIT-BIH AF database is commonly used in $\mathrm{AF}$ analysis because it includes about one million ECG beats with $43 \%$ classified as AF beats. It contains 25 ECGs of 10 hours recording lengths. Furthermore, it has two channel ambulatory ECG recordings in digital format with a $250 \mathrm{~Hz}$ sampling rate. The Challenge 2017 database [21] consists of 8528 recordings with 5154 records classified as Normal of 9 minutes recording lengths, 771 records classified as AF of 10 minutes recording lengths, 2557 records classified as other rhythm of 9 minutes recording lengths and 46 records classified as Noise and again of 9 minutes recording lengths. Signals were extracted from an AliveCor device at a sampling rate $300 \mathrm{~Hz}$. To match the MIT-BIH AF database the ECG recordings of the Challenge 2017 database were resampled to $250 \mathrm{~Hz}$.

\section{Methodology}

Template matching techniques have been widely used to identify similarities between waveforms. As described above, in AF rhythms, $\mathrm{P}$ waves either disappear or change to be appear like a sawtooth pattern. In this study, two templates called $\mathrm{P}$ presence and $\mathrm{P}$ absence were used to 
determine $\mathrm{P}$ wave presence or absence. The $\mathrm{P}$ presence template represented the normal $\mathrm{P}$ wave morphology as symbolized in figure 1 and identified from [22]. The $P$ wave absence template consisted of a sawtooth morphology and a disappear morphology generated from the MIT-BIH AF database. The both models were identified using probability based data adaptive techniques from nonconsecutive 10 minute traces, randomly selected, from each ECG record.

The standard deviation of five consecutive RR intervals was used to determine irregular RR intervals. The Standard deviation threshold (SDRR) was identified empirically based on RR intervals obtained from MIT-BIH AF database. Five consecutive RR intervals were selected following advice from senior cardiologists associated with the investigators.

\section{AF detection technique}

The presence of noise, especially in the baseline, leads to inaccurate detection of $\mathrm{P}$ wave presence or absence. Therefore, a real-time ECG reduction method reported by Allami et al. [23] was applied in order to suppress noise in the baseline. The AF detection method was implemented in four steps. First, the real-time QRS detection algorithm [24] was applied to extract six RR intervals. In the second step, five $\mathrm{P}$ waves were detected and the average correlation coefficient for $\mathrm{P}$ wave presence and absence computed. In the third step, the standard deviation of RR data (SDRR) was computed. Finally, if-then rules were applied to classify 5 consecutive beats as AF or non-AF episodes based on thresholding the calculated irregularity.

The algorithm for the detection of $\mathrm{AF}$ and non-AF episodes is as follows:

Input: Six consecutive ECG signals

Output: Classification of AF or non-AF

Step 1: Apply real-time ECG reduction method [23] to suppress noise from the baseline

Step 2: Apply real-time Pan and Tompkins [24] QRS detection algorithm to extract RR data from six ECG signals

Step 3: $S D R R=\sqrt{\frac{\sum_{i=1}^{5}(X i-\mu)^{2}}{5-1}}$

Step 4: For each beat compute the correlation coefficient (Corrcof) based on the $\mathrm{P}$ presence template (PP) and $\mathrm{P}$ absence template $(\mathrm{Pa})$

$\mathrm{Cpp}=\operatorname{Corrcof}(\mathrm{P}(\mathrm{i}), \mathrm{PP})$ where $\mathrm{i}=1,2, \ldots 5$

$\mathrm{r} 1=$ Average $(\mathrm{Cpp})$

if $(\mathrm{r} 1>0.80$ and $S D R R<0.059)$ then non AF Else $\mathrm{Cpa}=[\mathrm{Cpa} 1=\operatorname{Corrcof}(\mathrm{P}(\mathrm{i}), \mathrm{Pa} 1), \mathrm{Cpa} 2=$ Corrcof (P(i),Pa2) ]

r2= Max (Average $(\mathrm{Cpa} 1)$, Average $(\mathrm{Cpa} 2))$
Step 5: if $(\mathrm{r} 1<0.50$ and $\mathrm{r} 2>0.50$ and $S D R R>0.059)$ then AF Else unknown

\section{Results}

The performance of the suggested AF detection algorithm was quantified by calculation of sensitivity (Se), specificity ( $\mathrm{Sp}$ ) and accuracy (Ac) for each six ECG signals during 10 hours as described in formulas below

$$
\begin{aligned}
& \mathrm{Se}=\mathrm{TP} /(\mathrm{TP}+\mathrm{FN}) \times 100 \\
& \mathrm{Sp}=\mathrm{TN} /(\mathrm{TN}+\mathrm{FP}) \times 100 \\
& \mathrm{Ac}=(\mathrm{TN}+\mathrm{TP}) /(\mathrm{TP}+\mathrm{FP}+\mathrm{TN}+\mathrm{FN}) \times 100
\end{aligned}
$$

where True Positive (TP) refers to AF episodes correctly recognized, False Negative (FN) refers to missed $\mathrm{AF}$ episodes, False Positives (FP) refers to nonAF episodes recognized as AF's and True Negative (TN) refers to correctly recognized non-AF episodes.

Overall validation results of the proposed algorithm, including the results from the testing process based on the MIT-BIH Atrial Fibrillation database and generalization evaluation based on the Challenge 2017 database are depicted in Table 1.

Table 1. Overall performance in the testing databases of MIT-BIH AF database and the Challenge 2017 database.

\begin{tabular}{lrrl}
\hline \multicolumn{1}{c}{ Database } & $\mathrm{Se} \%$ & $\mathrm{Sp} \%$ & $\mathrm{Ac} \%$ \\
\hline MIT-BIH AF & 97.60 & 98.02 & 99 \\
Challenge 2017 & 95.27 & 90.60 & 96.80 \\
Other MIT-BIHAF [10] & 96.14 & 95.73 & 95.97 \\
\hline
\end{tabular}

The method advanced here incrementally displays better sensitivity, specificity and accuracy over recently published methods [ $8-11]$ of AF detection. The approach generalizes well to data sets not deployed in the training as observed by results from the (Challenge 2017) dataset.

\section{Conclusion}

The two template matching methods improved the detection accuracy by combining the similarity with irregular RR intervals results. Our results indicate that six consecutive ECG signals, are suffient to discern AF episodes from non-AF episodes with a high level of accuracy. The algorithm presented suits real-time cardiac monitoring as it requires only a few beats of ECG and is computationally simple. It is anticipated the proposed algorithm is clinically relevant since short AF episodes can be diagnosed in asymptomatic conditions. 


\section{Acknowledgements}

Ragheed Allami expresses his special thanks to the Ministry of Higher Education \& Scientific Research and The University of Technology in Iraq for supporting this work. The authors would like to thank the expert cardiologist Dr Saheb Al-Daher for his valuable recommendations, classifying ECG segments and providing specialist knowledge.

\section{References}

[1] Goldberger AL, Goldberger ZD, Shvilkin A. Clinical Electrocardiography: A simplified approach. Elsevier Health Sciences, 2017.

[2] Gacek A, Pedrycz W. ECG signal processing, classification and interpretation: a comprehensive framework of computational intelligence. Springer Science \& Business Media, 2011.

[3] Yochum M, Renaud C, Jacquir S. Automatic detection of P, QRS and T patterns in 12 leads ECG signal based on CWT. Biomedical Signal Processing and Control 2016;25: 46-52.

[4] Camm AJ, Kirchhof P, Lip GY, Schotten U, Savelieva I, Ernst $S$, et al. Guidelines for the management of atrial fibrillation: the Task Force for the management of atrial fibrillation of the European Society of Cardiology (ESC). European Heart Journal 2010;31(19): 2369-429.

[5] Colloca R. Implementation and testing of atrial fibrillation detectors for a mobile phone application. Thesis Politecnico Di Milano 2013.

[6] Savelieva I, Camm J. Update on atrial fibrillation: part I. Clinical Cardiology 2008;31(2): 55-62.

[7] Naccarelli GV, Varker H, Lin J, Schulman KL. Increasing prevalence of atrial fibrillation and flutter in the United States. The American Journal of Cardiology 2009;104(11): 1534-9.

[8] Carrara M, Carozzi L, Moss TJ, de Pasquale M, Cerutti S, Ferrario $\mathrm{M}$, et al. Heart rate dynamics distinguish among atrial fibrillation, normal sinus rhythm and sinus rhythm with frequent ectopy. Physiological Measurement 2015;36(9): 1873.

[9] Petrènas A, Marozas V, Sörnmo L. Low-complexity detection of atrial fibrillation in continuous long-term monitoring. Computers in Biology and Medicine 2015;65: 184-91.

[10] Zhou X, Ding H, Ung B, Pickwell-MacPherson E, Zhang Y. Automatic online detection of atrial fibrillation based on symbolic dynamics and Shannon entropy. Biomedical Engineering Online 2014;13(1): 18.

[11] Babaeizadeh S, Gregg RE, Helfenbein ED, Lindauer JM, Zhou SH. Improvements in atrial fibrillation detection for real-time monitoring. Journal of Electrocardiology 2009;42(6): 522-6.

[12] Oster J, Clifford GD. Impact of the presence of noise on RR interval-based atrial fibrillation detection. Journal of Electrocardiology 2015;48(6): 947-51.

[13] Li Q, Liu C, Oster J, Clifford GD. Signal processing and feature selection preprocessing for classification in noisy healthcare data. Machine Learning for Healthcare Technologies 2016;2: 33 .

[14] Couceiro R, Carvalho P, Henriques J, Antunes M, Harris M,
Habetha J, editors. Detection of atrial fibrillation using model-based ECG analysis. 19th International Conference on Pattern Recognition ICPR 2008: IEEE.

[15] Asgari S, Mehrnia A, Moussavi M. Automatic detection of atrial fibrillation using stationary wavelet transform and support vector machine. Computers in Biology and Medicine 2015;60: 132-42.

[16] Li P, Liu C, Wang X, Zheng D, Li Y, Liu C. A lowcomplexity data-adaptive approach for premature ventricular contraction recognition. Signal, Image and Video Processing 2014;8(1): 111-20.

[17] Seet RC, Friedman PA, Rabinstein AA. Prolonged rhythm monitoring for the detection of occult paroxysmal atrial fibrillation in ischemic stroke of unknown cause. Circulation 2011;124(4): 477-86.

[18] Flint AC, Banki NM, Ren X, Rao VA, Go AS. Detection of paroxysmal atrial fibrillation by 30 -day event monitoring in cryptogenic ischemic stroke. Stroke 2012;43(10): 2788-90.

[19] Rabinstein AA, Fugate JE, Mandrekar J, Burns JD, Seet RC, Dupont SA, et al. Paroxysmal atrial fibrillation in cryptogenic stroke: a case-control study. Journal of Stroke and Cerebrovascular Diseases 2013;22(8): 1405-11.

[20] Moody G. A new method for detecting atrial fibrillation using RR intervals. Computers in Cardiology 1983: 227-30.

[21] Chalellenge 2017 Database. Available from: https://www.physionet.org/physiobank/database/challenge/ 2017/training/ 2017.

[22] Laguna P, Mark RG, Goldberg A, Moody GB. A database for evaluation of algorithms for measurement of QT and other waveform intervals in the ECG. Computers in Cardiology; 1997: IEEE.

[23] Allami R, Stranieri A, Balasubramanian V, Jelinek HF, editors. ECG reduction for wearable sensor. 12th International Conference on Signal-Image Technology \& Internet-Based Systems (SITIS); 2016: IEEE.

[24] Pan J, Tompkins WJ. A real-time QRS detection algorithm. IEEE Transactions on Biomedical Engineering 1985(3): 230-6.

Address for correspondence:

Ragheed Allami

Federation University

University Drive, Mt Helen VIC 3350

Melbourne, Australia

ragheed.dawood@yahoo.com 